\title{
The Lorentzian Oscillator Group as a Geodesic Orbit Space
}

\author{
W. Batat, ${ }^{1, a)}$ P. M. Gadea, ${ }^{2, \text { b) }}$ and J. A. Oubiña ${ }^{3, c)}$ \\ ${ }^{1)}$ École Normale Supérieure d'Enseignement Technologique d'Oran, Département de Mathématiques et Informatique, \\ B.P. 1523, El M'Naouar, Oran, Algeria \\ 2) Instituto de Física Fundamental, CSIC, Serrano 113-bis, 28006-Madrid, Spain \\ ${ }^{3)}$ Departamento de Xeometría e Topoloxía, Facultade de Matemáticas, Universidade de Santiago de Compostela, \\ 15782-Santiago de Compostela, Spain
}

(Dated: 4 June 2012)

We prove that the four-dimensional oscillator group Os endowed with any of its usual left-invariant Lorentzian metrics, is a Lorentzian geodesic (so in particular null-geodesic) orbit space with some of its homogeneous descriptions, corresponding to certain homogeneous Lorentzian structures. Each time that Os is endowed with a suitable metric and an appropriate homogeneous Lorentzian structure, it is a candidate for constructing solutions in eleven-dimensional supergravity with at least twenty-four of the thirty-two possible supersymmetries.

PACS numbers: 02.20. Qs, 04.65,+e

Keywords: Lorentzian oscillator group, geodesic orbit space, null-geodesic orbit space, homogeneous Lorentzian structures, eleven-dimensional supergravity

\section{INTRODUCTION}

The 4-dimensional oscillator group was named so by Streater $^{26}$, because its Lie algebra is associated to the harmonic oscillator problem. This is the lowestdimensional member of a family of Lie groups called the oscillator groups (see for instance Medina and Revoy ${ }^{20}$, Bromberg and Medina ${ }^{4}$, and Ref. ${ }^{15}$ ) but we restrict ourselves in the present paper to the 4-dimensional one and denote it by Os.

This group has many properties useful both in geometry and physics. To mention but two geometrical applications, Medina ${ }^{19}$ proved that Os is the only fourdimensional non-Abelian simply-connected solvable Lie group which admits a bi-invariant Lorentzian metric; and Console, Ovando and Subils ${ }^{7}$ obtain solvable models for Kodaira surfaces by using suitable lattices on the oscillator group. We also recall four physical applications: Firstly, Nappi and Witten ${ }^{23}$ showed that it is an example of homogeneous 4-dimensional spacetime (called in the literature Nappi-Witten group or space) that substitutes for 4-dimensional Minkowski space from an ungauged WZW model, and it is viewed as a four-dimensional monochromatic plane wave. Secondly, Bromberg and Medina ${ }^{3}$ proved that with a suitable left-invariant metric (which coincides with the bi-invariant metric $g_{0}$ that we consider in this paper), it becomes a spacetime with singularities in the sense of Hawking and Penrose. Thirdly, it furnishes exact solutions of the Einstein-Yang-Mills equations (Levichev ${ }^{17}$, see also Ref. ${ }^{8}$ ). Finally, Boucetta and Medina ${ }^{2}$ have proved that it provides solutions of the classical Yang-Baxter equations.

\footnotetext{
a) batatwafa@yahoo.fr

b) pmgadea@iff.csic.es

c) ja.oubina@usc.es
}

On the other hand, as is well known, É. Cartan ${ }^{6}$ characterised locally symmetric spaces as those Riemannian manifolds $(M, g)$ with parallel curvature tensor, $\nabla R=0$, where $\nabla$ denotes the Levi-Civita connection of $g$. This characterisation was extended by Ambrose and Singer ${ }^{1}$ to homogeneous Riemannian spaces, as those Riemannian manifolds admitting a homogeneous Riemannian structure $S$, that is, a $(1,2)$ tensor field satisfying the usually called Ambrose-Singer equations (see Eqs. (3) below). This was extended to the pseudo-Riemannian case in Ref. ${ }^{13}$. In the present paper we apply some results on homogeneous (Lorentzian) structures to solve the problem under study.

The aim of this paper is to give another physical application: To know when a homogeneous presentation of Os is a homogeneous Lorentzian space all of whose null-geodesics are homogeneous, so being (see Remark III.4 below) a candidate for constructing solutions in 11-dimensional supergravity that preserve more than 24 of the available 32 supersymmetries. According to Figueroa-O'Farrill, Meessen and Philip ${ }^{11,12}$ and Meessen $^{22}$, this general fact follows because all Penrose limits (which are known to preserve the amount of supersymmetry), of such a solution must preserve homogeneity, and this is ensured if all the null-geodesics are homogeneous (Philip ${ }^{25}$ ).

To this end we find the homogeneous geodesics for each of the usual homogeneous descriptions of the Lie group Os endowed with the left-invariant Lorentzian metrics $g_{\varepsilon},-1<\varepsilon<1$, (see $\S \mathrm{IIC}$ ) and so determine in which cases $\left(\mathrm{Os}, g_{\varepsilon}\right.$ ) is (see Definition II.2 below) a Lorentzian geodesic orbit space, so in particular a Lorentzian nullgeodesic orbit space, and which are then the corresponding geometric types of homogeneous Lorentzian structures.

As for the contents, in $\S I I$ we give some preliminaries. In $\S$ III we prove Theorems III.1 and III.2, obtaining all the geodesic vectors of the different homogeneous 
descriptions of the Lorentzian Lie groups $\left(\mathrm{Os}, g_{\varepsilon}\right)$ determined by their homogeneous Lorentzian structures, and in Theorem III.3 we give the homogeneous descriptions (in terms of the homogeneous Lorentzian structures) with which Os is a Lorentzian geodesic orbit space, showing that it is never a null-geodesic orbit space which is not a geodesic orbit space. Then we give Corollary III.6, exhibiting the corresponding geometric types.

\section{PRELIMINARIES}

\section{A. Homogeneous geodesics}

We now recall some definitions on homogeneous geodesics.

Let $(M=G / H, g)$ be a reductive homogeneous pseudo-Riemannian space with reductive decomposition $\mathfrak{g}=\mathfrak{h}+\mathfrak{m}$ (direct sum), where $\mathfrak{g}$ and $\mathfrak{h}$ denote the Lie algebras of $G$ and $H$ and $\mathfrak{m}$ stands for the corresponding $\operatorname{Ad}(H)$-invariant complement to $\mathfrak{h}$. Let $o \in M$ be a fixed point. A nontrivial geodesic $\gamma$ in $M=G / H$ is said to be a homogeneous geodesic if it is the orbit of a one-parameter subgroup of $G$. By homogeneity one can suppose that the geodesics pass through the base point $o$. Such homogeneous geodesics can always be reparameterised so that they are given by

$$
\gamma(t)=\exp (t W) \cdot o
$$

for some vector $W \in \mathfrak{g}=\mathfrak{h}+\mathfrak{m}$ such that $W_{\mathfrak{m}} \neq 0$, where the subscript $\mathfrak{m}$ denotes projection on $\mathfrak{m}$, and such a vector $W$ is called a geodesic vector. If $\gamma^{\prime}$ has nonzero norm, this is equivalent to the condition $\nabla_{\gamma^{\prime}} \gamma^{\prime}=0$, where $\nabla$ stands for the Levi-Civita connection, but for null geodesics, this condition can be relaxed to $\nabla_{\gamma^{\prime}} \gamma^{\prime}=$ $f(\gamma) \gamma^{\prime}$, for certain function $f(\gamma)$. It follows from the usual Koszul formula for the Levi-Civita connection that a vector $W \in \mathfrak{g}$ such that $W_{\mathfrak{m}} \neq 0$ is geodesic if and only if

$$
\left\langle[W, Z]_{\mathfrak{m}}, W_{\mathfrak{m}}\right\rangle=k\left\langle W_{\mathfrak{m}}, Z\right\rangle
$$

for all $Z \in \mathfrak{m}$ and some $k \in \mathbb{R}$ depending on $W_{\mathfrak{m}},\langle$, being the scalar product on $\mathfrak{m}$ defined by the pseudoRiemannian metric $g$ under the usual identification $\mathfrak{m} \equiv$ $T_{o} M$.

Remark II.1. If a geodesic is homogeneous with respect to a homogeneous description $M=G / H$ then it is not necessarily homogeneous with respect to other possible description of $M$ as a reductive homogeneous pseudoRiemannian space (see Ref. ${ }^{10}$ ).

According to Defs. 16 and 37 in Dušek ${ }^{9}$ (see also Def. 1 in Meessen $\left.^{22}\right)$, and denoting by $I_{0}(M, g)$ the identity component of the full isometry group of a Riemannian manifold $(M, g)$, we have the next definition.
Definition II.2. A reductive homogeneous pseudoRiemannian space $(M=G / H, g)$, with a fixed reductive decomposition $\mathfrak{g}=\mathfrak{h}+\mathfrak{m}$, is said to be a g.o. space if each nontrivial geodesic $\gamma$ in $M=G / H$ is homogeneous (that is, $\gamma$ is of the form (1) with $W \in \mathfrak{g}$ satisfying (2)), and a n.g.o. space if all its null-geodesics are homogeneous (in both cases, g.o. means as usual "geodesic orbit"). A homogeneous pseudo-Riemannian manifold $(M, g)$ is called a g.o. manifold if $M=G / H$ is a g.o. space for $G=I_{0}(M, g)$.

\section{B. Homogeneous pseudo-Riemannian structures}

Let $(M, g)$ be a connected pseudo-Riemannian manifold of dimension $n$ and signature $(s, n-s)$. Let $\nabla$ be the Levi-Civita connection of $g$ and $R$ the curvature tensor field. A homogeneous pseudo-Riemannian structure on $(M, g)$ is a tensor field $S$ of type $(1,2)$ on $M$ such that the connection $\widetilde{\nabla}=\nabla-S$ satisfies the Ambrose-Singer Eqs.

$$
\widetilde{\nabla} g=0, \quad \widetilde{\nabla} R=0, \quad \widetilde{\nabla} S=0
$$

If $g$ is a Lorentzian metric (that is, $s=1$ ), we say that $S$ is a homogeneous Lorentzian structure. If $(M, g)$ is connected, simply-connected and geodesically complete, then (cf. Ref. ${ }^{13}$ ) it admits a homogeneous pseudoRiemannian structure if and only if it is a reductive homogeneous pseudo-Riemannian manifold.

A homogeneous pseudo-Riemannian structure $S$ on a connected, simply-connected and geodesically complete pseudo-Riemannian manifold $(M, g)$ defines a reductive decomposition of a certain homogeneous description of $M$ in the following way. We fix a point $o \in M$ and consider $\mathfrak{m}=T_{o}(M)$. The holonomy algebra $\mathfrak{h}$ of $\widetilde{\nabla}$ is the Lie subalgebra of the Lie algebra of skew-symmetric endomorphisms of $(\mathfrak{m},\langle\rangle$,$) generated by the curvature$ operators $\widetilde{R}_{Z Z^{\prime}}$ of the connection $\widetilde{\nabla}=\nabla-S$, where $Z, Z^{\prime} \in \mathfrak{m}$. Then $\left(\right.$ Nomizu $^{24}$, see also Refs. $\left.{ }^{1,27}\right)$ a Lie bracket is defined in the vector space direct sum $\mathfrak{g}=\mathfrak{h}+\mathfrak{m}$ by

$$
\begin{aligned}
{[U, V] } & =U V-V U \\
{[U, Z] } & =U(Z) \\
{\left[Z, Z^{\prime}\right] } & =\widetilde{R}_{Z Z^{\prime}}+S_{Z} Z^{\prime}-S_{Z^{\prime}} Z
\end{aligned}
$$

for all $U, V \in \mathfrak{h}, Z, Z^{\prime} \in \mathfrak{m}$. The connected simplyconnected Lie group $G^{\prime}$ generated by $\mathfrak{g}$ acts transitively on $M$ via isometries and $M \equiv G^{\prime} / H^{\prime}$, where $H^{\prime}$ is the connected Lie subgroup of $G^{\prime}$ generated by $\mathfrak{h}$. The set $\Gamma$ of elements of $G^{\prime}$ which act trivially on $M$ is a discrete normal subgroup of $G^{\prime}$ contained in $H^{\prime}$, and if we set $G=G^{\prime} / \Gamma$ and $H=H^{\prime} / \Gamma$, then $G / H$ is a homogeneous presentation of $M$ such that the Lie group $G$ acts transitively and effectively on $M$ as a group of isometries, with isotropy subgroup $H$. Then $\mathfrak{g}=\mathfrak{h}+\mathfrak{m}$ is said to 
be the reductive decomposition associated to the homogeneous pseudo-Riemannian structure $S$, and $\widetilde{\nabla}$ is called the canonical connection of this reductive decomposition.

If $W$ in equation (2) belongs to $\mathfrak{m}$, then the geodesic $\gamma$ given by (1) is called a canonically homogeneous geodesic, since then it is also a geodesic for the canonical connection.

There are some pseudo-Riemannian manifolds all of whose geodesics are homogeneous, as naturally reductive pseudo-Riemannian spaces and in particular pseudo-Riemannian symmetric spaces; actually, for such spaces every geodesic is canonically homogeneous, with respect to naturally reductive and symmetric decompositions, respectively (see Ch. $10 \S 3$ and Ch. $11 \S 3$ in Ref. ${ }^{16}$ ).

We consider now a real vector space $V$ endowed with a scalar product $\langle$,$\rangle of signature (s, n-s)$, which is the model for each tangent space $T_{p} M, p \in M$, of a (reductive homogeneous) pseudo-Riemannian manifold of signature $(s, n-s)$. Consider the vector space $\mathcal{S}(V)$ of tensors of type $(0,3)$ on $(V,\langle\rangle$,$) satisfying the same$ symmetries as a homogeneous pseudo-Riemannian structure $S$, that is, as it easily follows from the the first Ambrose-Singer Eq. in (3), $\mathcal{S}(V)=\left\{S \in \otimes^{3} V^{*}\right.$ : $\left.S_{X Y Z}=-S_{X Z Y}, X, Y, Z \in V\right\}$, where $S_{X Y Z}=$ $\left\langle S_{X} Y, Z\right\rangle$. The scalar product on $V$ induces in a natural way a scalar product on $\mathcal{S}(V)$, given by $\left\langle S, S^{\prime}\right\rangle=$ $\sum_{i, j, k=1}^{n} \varepsilon_{i} \varepsilon_{j} \varepsilon_{k} S_{e_{i} e_{j} e_{k}} S_{e_{i} e_{j} e_{k}}^{\prime}$, where $\left\{e_{i}\right\}$ is an orthonormal basis of $V$, that is, $\left\langle e_{i}, e_{i}\right\rangle=\varepsilon_{i}$, with $\varepsilon_{i}=-1$ if $1 \leq i \leq s$ and $\varepsilon_{i}=1$ if $s+1 \leq i \leq n$. Let $c_{12}: \mathcal{S}(V) \rightarrow V^{*}$ be the map defined by

$$
c_{12}(S)(Z)=\sum_{i=1}^{n} \varepsilon_{i} S_{e_{i} e_{i} Z}, \quad Z \in V .
$$

The decomposition of the space $\mathcal{S}(V)$ into invariant and irreducible subspaces under the action of the pseudoorthogonal group $O(s, n-s)$ defined by $(a S)_{X Y Z}=$ $S_{a^{-1} X a^{-1} Y a^{-1} Z}, a \in O(s, n-s)$, is given, denoting by $\mathfrak{S}_{X Y Z} S_{X Y Z}$ the cyclic sum of $S_{X Y Z}$ with respect to $X, Y, Z$, by

Theorem II.3. ( $\left.{ }^{14}\right)$ If $\operatorname{dim} V \geq 3$, the space $\mathcal{S}(V)$ decomposes into the orthogonal direct sum of subspaces which are invariant and irreducible under the action of
$O(s, n-s)$, as

$$
\mathcal{S}(V)=\mathcal{S}_{1}(V) \oplus \mathcal{S}_{2}(V) \oplus \mathcal{S}_{3}(V),
$$

where

$$
\begin{aligned}
\mathcal{S}_{1}(V)=\left\{S \in \mathcal{S}(V): S_{X Y Z}=\langle X, Y\rangle \theta(Z)\right. & \left.-\langle X, Z\rangle \theta(Y), \theta \in V^{*}\right\}, \\
& -\left\langle\mathcal{S}_{X Y Z} S_{X Y Z}=0, c_{12}(S)=0\right\}, \\
\mathcal{S}_{2}(V)=\left\{S \in \mathcal{S}(V): \mathcal{S}_{X}\right) & \mathcal{S}_{3}(V)=\left\{S \in \mathcal{S}(V): S_{X Y Z}+S_{Y X Z}=0\right\} .
\end{aligned}
$$

If $\operatorname{dim} V=2$ then $\mathcal{S}(V)=\mathcal{S}_{1}(V)$.

It is then immediate that

$$
\mathcal{S}_{2}(V) \oplus \mathcal{S}_{3}(V)=\left\{S \in \mathcal{S}(V): c_{12}(S)=0\right\} .
$$

\section{Homogeneous Lorentzian structures on the oscillator group}

We now recall some definitions, and results on the homogeneous Lorentzian structures, on the oscillator group.

The 4-dimensional oscillator group $\mathrm{Os}$ is the connected simply-connected Lie group with Lie algebra $\mathfrak{o s}=$ $\langle\{P, X, Y, Q\}\rangle$ having $\left(\right.$ Streater $^{26}$ ) nonzero brackets

$$
[X, Y]=P, \quad[Q, X]=Y, \quad[Q, Y]=-X .
$$

It will be endowed with a left-invariant Lorentzian metric $g_{\varepsilon}$, with $-1<\varepsilon<1$, having nonzero scalar products $\langle,\rangle_{\varepsilon}$ on os of the elements $P, X, Y, Q$, given by

$$
\begin{gathered}
\langle P, P\rangle_{\varepsilon}=\langle Q, Q\rangle_{\varepsilon}=\varepsilon, \\
\langle P, Q\rangle_{\varepsilon}=\langle X, X\rangle_{\varepsilon}=\langle Y, Y\rangle_{\varepsilon}=1 .
\end{gathered}
$$

If $\varepsilon=0$, the corresponding Lorentzian metric $g_{0}$ is also right-invariant. If $\varepsilon \neq 0$, the metric $g_{\varepsilon}$ is not bi-invariant.

We shall denote by $\{\eta, \alpha, \beta, \xi\}$ the basis of differential 1-forms dual to $\{P, X, Y, Q\}$. We have

Theorem II.4. $\left({ }^{8}\right)$ The homogeneous Lorentzian structures on $\left(\mathrm{Os}, g_{0}\right)$ are the following:

(i) $S_{(x, y, z, w)}=\left(x \eta+x y \alpha+x z \beta+\left(w+\frac{1}{2}\right) \xi\right) \otimes(\alpha \wedge \beta)-\left(x z \eta+x y z \alpha+\left(x z^{2}+\frac{1}{2}\right) \beta+z w \xi\right) \otimes(\alpha \wedge \xi)$

$$
+\left(x y \eta+\left(x y^{2}+\frac{1}{2}\right) \alpha+x y z \beta+y w \xi\right) \otimes(\beta \wedge \xi), \quad x, y, z, w \in \mathbb{R}, \quad x \neq 0 ;
$$

(ii) $S_{(q, c)}=\frac{1}{2} \xi \otimes(\alpha \wedge \beta)-\frac{1}{2} \beta \otimes(\alpha \wedge \xi)+(q \alpha+c \xi) \otimes(\beta \wedge \xi), \quad q, c \in \mathbb{R}, \quad q \neq \frac{1}{2}$;

(iii) $S_{(b, c)}=\frac{1}{2} \xi \otimes(\alpha \wedge \beta)+\left(b \xi-\frac{1}{2} \beta\right) \otimes(\alpha \wedge \xi)+\left(\frac{1}{2} \alpha+c \xi\right) \otimes(\beta \wedge \xi), \quad b, c \in \mathbb{R} ;$

(iv) $S_{(h, t, b)}=\frac{1}{2} \xi \otimes(\alpha \wedge \beta)+\left(h \alpha+\left(t-\frac{1}{2}\right) \beta+b \xi\right) \otimes(\alpha \wedge \xi)+\left(\left(\frac{1}{2}-\frac{h^{2}}{t}\right) \alpha-h \beta-\frac{h b}{t} \xi\right) \otimes(\beta \wedge \xi)$,

$$
h, t, b \in \mathbb{R}, \quad t \neq 0
$$

(v) $S_{(a, b, c)}=a \xi \otimes(\alpha \wedge \beta)+\left(b \xi-\frac{1}{2} \beta\right) \otimes(\alpha \wedge \xi)+\left(\frac{1}{2} \alpha+c \xi\right) \otimes(\beta \wedge \xi), \quad a, b, c \in \mathbb{R}, \quad a \neq \frac{1}{2} ;$

(vi) $S_{a}=a(\xi \otimes(\alpha \wedge \beta)-\beta \otimes(\alpha \wedge \xi)+\alpha \otimes(\beta \wedge \xi)), \quad a \in \mathbb{R}, \quad a \neq \frac{1}{2}$. 
As for $\left(\mathrm{Os}, g_{\varepsilon}\right)$, we have

Theorem II.5. $\left.{ }^{15}\right)$ The homogeneous Lorentzian structures on $\left(\mathrm{Os}, g_{\varepsilon}\right)$, with $-1<\varepsilon<1, \varepsilon \neq 0$, are given by

$$
\begin{gathered}
S_{(a, b)}=\frac{\varepsilon}{2} \beta \otimes(\eta \wedge \alpha)-\frac{\varepsilon}{2} \alpha \otimes(\eta \wedge \beta)-\frac{1}{2} \beta \otimes(\alpha \wedge \xi) \\
+\frac{1}{2} \alpha \otimes(\beta \wedge \xi)+(a \eta+b \xi) \otimes(\alpha \wedge \beta), \quad a, b \in \mathbb{R} .
\end{gathered}
$$

\section{THE OSCILLATOR GROUP AS A LORENTZIAN GEODESIC ORBIT SPACE}

The oscillator group Os is a connected and simply connected manifold, and every Lorentzian metric $g_{\varepsilon}$, $-1<\varepsilon<1$, is geodesically complete (see Refs. ${ }^{17,18}$ ). Then every homogeneous Lorentzian structure $S$ on $\left(\mathrm{Os}, g_{\varepsilon}\right)$ has an associated reductive decomposition $\mathfrak{g}=$ $\mathfrak{h}+\mathfrak{m}$, with $\mathfrak{m}=T_{0}(\mathrm{Os}) \equiv \mathfrak{o s}$, and a corresponding homogeneous presentation $G / H$, where $G \subseteq I_{0}\left(\mathrm{Os}, g_{\varepsilon}\right)$ acts transitively on Os.

In order to know when the oscillator group, with each one of the homogeneous presentations so determined, is a Lorentzian g.o. space, we now find the homogeneous geodesics for the reductive decompositions associated to the homogeneous Lorentzian structures recalled in Theorems II.4 and II.5 above. As is well known (cf. for instance Ref. ${ }^{5}$, p. 315) and we recalled in Subsection II A, this amounts to find, for each reductive decomposition $\mathfrak{g}=\mathfrak{h}+\mathfrak{m}$, the geodesic vectors, that is, the vectors $W \in \mathfrak{g}$ having nonzero projection to $\mathfrak{m}$ and satisfying equation (2).

We have

Theorem III.1. For each homogeneous Lorentzian structure $S$ on the oscillator group $\left(\mathrm{Os}, g_{0}\right)$ given in Theorem II.4, let $\mathfrak{g}=\mathfrak{h}+\mathfrak{m}$ be the reductive decomposition associated to $S$ and let $G / H$ be the corrresponding homogeneous presentation of $\mathrm{Os}, G \subseteq I_{0}\left(\mathrm{Os}, g_{0}\right)$. The set of geodesic vectors through the origin $o \in \mathrm{Os}=G / H$ is given in Table I, where the first column indicates the case of Theorem II.4 to which each homogeneous Lorentzian structure $S$ on $\left(\mathrm{Os}, g_{0}\right.$ ) corresponds (and where both cases (i) and (vi) are broken into two subcases according to the dimension of the holonomy algebra $\mathfrak{h}$ of the canonical connection $\tilde{\nabla}=\nabla-S)$. Furthermore, all geodesics are canonically homogeneous only for the cases iii (for $(b, c)=(0,0))$ and $\mathbf{v i}$.

TABLE I. Geodesic vectors $W \in \mathfrak{g}=\mathfrak{h}+\mathfrak{m}$ of $\left(\mathrm{Os}=G / H, g_{0}\right)$, $\mathfrak{m}=\langle\{P, X, Y, Q\}\rangle$

\begin{tabular}{|r|l|c|l|}
\hline & $S$ & $\operatorname{dim} \mathfrak{h}$ & Geodesic vectors \\
\hline $\mathbf{i}_{1}$ & $\begin{array}{c}S_{(x, y, z, w)}, x \neq 0 \\
(y, z, w) \neq(0,0,0)\end{array}$ & 1 & $\begin{array}{l}W=\left(\lambda_{1} x+\lambda_{2} x y+\lambda_{3} x z+\lambda_{4} w\right) U+\lambda_{1} P+\lambda_{2} X+\lambda_{3} Y+\lambda_{4} Q \\
W=\mu U+\lambda_{1} P+\lambda_{4}(y X+z Y+Q)\end{array}$ \\
\hline $\mathbf{i}_{2}$ & $S_{(x, 0,0,0), x \neq 0}$ & 0 & $\begin{array}{l}W=\lambda_{2} X+\lambda_{3} Y+\lambda_{4} Q \\
W=\lambda_{1} P+\lambda_{4} Q\end{array}$ \\
\hline $\mathbf{i i}$ & $S_{(q, c), q \neq \frac{1}{2}}$ & 1 & $\begin{array}{l}W=\mu U+\lambda_{1} P+\lambda_{2} X \\
W=\left(\left(\frac{1}{2}-q\right) \lambda_{2}-c \lambda_{4}\right) U+\lambda_{1} P+\lambda_{2} X+\lambda_{3} Y+\lambda_{4} Q\end{array}$ \\
\hline $\mathbf{i i i}$ & $S_{(b, c)}$ & 0 & $\begin{array}{l}W=\lambda_{1} P+\lambda_{2} X+\lambda_{3} Y \quad \text { if }(b, c) \neq(0,0) \\
W=\lambda_{1} P+\lambda_{2} X+\lambda_{3} Y+\lambda_{4} Q, \quad \text { if }(b, c)=(0,0)\end{array}$ \\
\hline $\mathbf{i v}$ & $S_{(h, t, b)}, t \neq 0$ & 1 & $\begin{array}{l}W=\mu U+\lambda_{1} P+\left(\lambda_{3} h / t\right) X+\lambda_{3} Y \\
W=t^{-1}\left(\lambda_{2} h+\lambda_{3} t+\lambda_{4} b\right) U+\lambda_{1} P+\lambda_{2} X+\lambda_{3} Y+\lambda_{4} Q\end{array}$ \\
\hline $\mathbf{v}$ & $S_{(a, b, c)}, a \neq \frac{1}{2}$ & 0 & $\begin{array}{l}W=\lambda_{1} P+\lambda_{2} X+\lambda_{3} Y \\
W=\lambda_{1} P+\lambda_{4}\left(a-\frac{1}{2}\right)^{-1}(c X-b Y)+\lambda_{4} Q\end{array}$ \\
\hline $\mathbf{v i}$ & $S_{a}, a \neq \pm \frac{1}{2}$ & 2 & $\begin{array}{l}W=\lambda_{1} P+\lambda_{2} X+\lambda_{3} Y+\lambda_{4} Q \\
W=\mu U+\nu V+\lambda_{1} P+\lambda_{2} X+\lambda_{3} Y, \quad \text { with } \mu \lambda_{2}+\nu \lambda_{3}=0\end{array}$ \\
\hline $\mathbf{v i} \mathbf{i}_{2}$ & $S_{-\frac{1}{2}}$ & 0 & $W=\lambda_{1} P+\lambda_{2} X+\lambda_{3} Y+\lambda_{4} Q$ \\
\hline
\end{tabular}

Proof. Equations (4) give the Lie brackets of each possi-

ble reductive decomposition for the manifold (Os, $\left.g_{0}\right)$ de- 
fined by the homogeneous Lorentzian structures in each case of Theorem II.4 (cf. Ref. ${ }^{8}, \S 3$ ). Then, we have the following possibilities, where each $\mathfrak{h}$ is the holonomy algebra, generated by the curvature operators $\widetilde{R}_{Z Z^{\prime}}$ $\left(Z, Z^{\prime} \in \mathfrak{m} \equiv \mathfrak{o s}\right)$ of the connection $\widetilde{\nabla}=\nabla-S$ associated to each homogeneous Lorentzian structure $S$.

CASE $\mathbf{i}(x, y, z, w \in \mathbb{R}, x \neq 0)$. The holonomy algebra $\mathfrak{h}$ is generated by $U \in \operatorname{End}(\mathfrak{m})$ such that $\widetilde{R}_{X Y}=w U$, $\widetilde{R}_{Q X}=x z U, \widetilde{R}_{Q Y}=-x y U$. We consider two subcases.

SubCASE $\mathbf{i}_{\mathbf{1}}$. If $y, z, w$ are not all null, the reductive decomposition associated to the structure $S_{(x, y, z, w)}$ is given by $\mathfrak{g}_{(x, y, z, w)}=\mathfrak{h}+\mathfrak{m}=\langle\{U, P, X, Y, Q\}\rangle$, with not always null brackets

$$
\begin{gathered}
{[U, X]=z P-Y, \quad[U, Y]=-y P+X,} \\
{[U, Q]=-z X+y Y, \quad[P, X]=-x z P+x Y,} \\
{[P, Y]=x y P-x X, \quad[P, Q]=x z X-x y Y,} \\
{[X, Y]=w U+\left(x\left(y^{2}+z^{2}\right)+1\right) P-x y X-x z Y,} \\
{[X, Q]=-x z U+z w P+x y z X-\left(w+x y^{2}+1\right) Y,} \\
{[Y, Q]=x y U-y w P+\left(w+x z^{2}+1\right) X-x y z Y .}
\end{gathered}
$$

Then a vector $W=\mu U+\lambda_{1} P+\lambda_{2} X+\lambda_{3} Y+\lambda_{4} Q \in$ $\mathfrak{g}_{(x, y, z, w)}$ is a geodesic vector if and only if (2) holds. We set $Z=P, X, Y$ and $Q$ in (2), and we find that (2) is equivalent to the following system of equations,

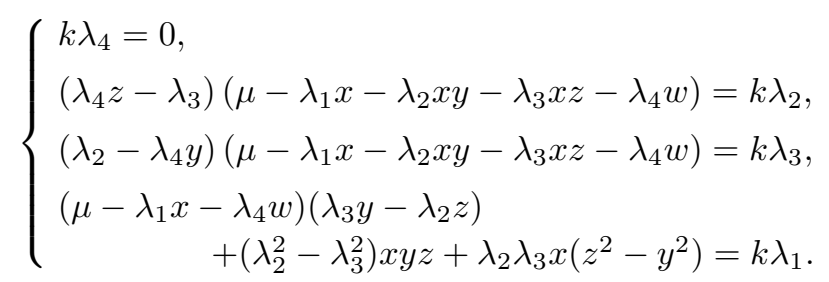

For $k \neq 0$ we have $W_{\mathfrak{m}}=0$. For $k=0$, the fourth equation follows from the second and the third, and we have that either $\mu=\lambda_{1} x+\lambda_{2} x y+\lambda_{3} x z+\lambda_{4} w$ or $\lambda_{2}=\lambda_{4} y$ and $\lambda_{3}=\lambda_{4} z$. Then the geodesic vectors have one of the following forms,

$$
\begin{aligned}
W= & \left(\lambda_{1} x+\lambda_{2} x y+\lambda_{3} x z+\lambda_{4} w\right) U \\
& +\lambda_{1} P+\lambda_{2} X+\lambda_{3} Y+\lambda_{4} Q, \\
W= & \mu U+\lambda_{1} P+\lambda_{4}(y X+z Y+Q) .
\end{aligned}
$$

SubCASE $\mathbf{i}_{2}$. If $y=z=w=0$, the holonomy algebra $\mathfrak{h}$ is trivial and the reductive decomposition associated to $S_{(x, 0,0,0)}$ is $\mathfrak{g}_{x}=\{0\}+\mathfrak{m}=\langle\{P, X, Y, Q\}\rangle$, with nonvanishing brackets

$$
\begin{gathered}
{[P, X]=x Y, \quad[P, Y]=-x X, \quad[X, Y]=P,} \\
{[X, Q]=-Y, \quad[Y, Q]=X .}
\end{gathered}
$$

Then $W=\lambda_{1} P+\lambda_{2} X+\lambda_{3} Y+\lambda_{4} Q \in \mathfrak{g}_{x}$ is geodesic if and only if

$$
\left\{\begin{array}{l}
k \lambda_{1}=k \lambda_{4}=0, \\
\lambda_{1} \lambda_{3} x=k \lambda_{2}, \\
\lambda_{1} \lambda_{2} x=-k \lambda_{3} .
\end{array}\right.
$$

that is, $W \in \mathfrak{g}_{x}$ is of one of the forms

$$
W=\lambda_{2} X+\lambda_{3} Y+\lambda_{4} Q, \quad W=\lambda_{1} P+\lambda_{4} Q .
$$

CAsE ii $\left(q, c \in \mathbb{R}, q \neq \frac{1}{2}\right)$. The holonomy algebra is $\mathfrak{h}=\langle\{U\}\rangle$, where $U=\left(q-\frac{1}{2}\right)^{-1} \widetilde{R}_{Q Y}$, and the reductive decomposition associated to $S_{(q, c)}$ is $\mathfrak{g}_{(q, c)}=\mathfrak{h}+\mathfrak{m}=$ $\langle\{U, P, X, Y, Q\}\rangle$, with not always null brackets

$$
\begin{gathered}
{[U, Y]=P, \quad[U, Q]=-Y, \quad[X, Y]=\left(q+\frac{1}{2}\right) P,} \\
{[X, Q]=-\left(q+\frac{1}{2}\right) Y, \quad[Y, Q]=-\left(q-\frac{1}{2}\right) U-c P+X .}
\end{gathered}
$$

Then $W=\mu U+\lambda_{1} P+\lambda_{2} X+\lambda_{3} Y+\lambda_{4} Q \in \mathfrak{g}_{(q, c)}$ is geodesic if and only if it satisfies (2), that is,

$$
\left\{\begin{array}{l}
k \lambda_{2}=k \lambda_{4}=0, \\
\lambda_{4}\left[\mu+\lambda_{2}\left(q-\frac{1}{2}\right)+\lambda_{4} c\right]=k \lambda_{3}, \\
\lambda_{3}\left[\mu+\lambda_{2}\left(q-\frac{1}{2}\right)+\lambda_{4} c\right]=-k \lambda_{1} .
\end{array}\right.
$$

For $k \neq 0$ we only have $W=0$, and for $k=0$ we get that $W \in \mathfrak{g}_{(q, c)}$ is a geodesic vector if and only if it has one of the forms

$$
\begin{aligned}
& W=\mu U+\lambda_{1} P+\lambda_{2} X, \\
& W=\left(\left(\frac{1}{2}-q\right) \lambda_{2}-c \lambda_{4}\right) U+\lambda_{1} P+\lambda_{2} X+\lambda_{3} Y+\lambda_{4} Q .
\end{aligned}
$$

CASE iii $(b, c \in \mathbb{R})$. The holonomy algebra $\mathfrak{h}$ is 0 and the reductive decomposition associated to $S_{(b, c)}$ is $\mathfrak{g}_{(b, c)}=\{0\}+\mathfrak{m}=\langle\{P, X, Y, Q\}\rangle$, with non-null brackets

$$
[X, Y]=P, \quad[X, Q]=-b P-Y, \quad[Y, Q]=-c P+X
$$

Then $W=\lambda_{1} P+\lambda_{2} X+\lambda_{3} Y+\lambda_{4} Q \in \mathfrak{g}_{(b, c)}$ is geodesic if and only if it satisfies (2), that is,

$$
\left\{\begin{array}{l}
k \lambda_{4}=0, \\
b \lambda_{4}^{2}=k \lambda_{2}, \\
c \lambda_{4}^{2}=k \lambda_{3}, \\
\lambda_{4}\left(b \lambda_{2}+c \lambda_{3}\right)=-k \lambda_{1} .
\end{array}\right.
$$

For $k \neq 0$ these equations give $W=0$. For $k=0$, if $(b, c) \neq(0,0)$ then $\lambda_{4}=0$ and all geodesic vectors $W \in \mathfrak{g}_{(b, c)}$ are of the form $W=\lambda_{1} P+\lambda_{2} X+\lambda_{3} Y$, and in the remaining case $(b, c)=(0,0)$, all vectors $W \in \mathfrak{g}_{(0,0)}$ are geodesic. 
CASE iv $(h, t, b \in \mathbb{R}, t \neq 0)$. The holonomy algebra $\mathfrak{h}$ is generated by $U=\widetilde{R}_{Q X}$, as also $\widetilde{R}_{Q Y}=-(h / t) U$. The reductive decomposition associated to $S_{(h, t, b)}$ is $\mathfrak{g}_{(h, t, b)}=$ $\mathfrak{h}+\mathfrak{m}=\langle\{U, P, X, Y, Q\}\rangle$, with not always null brackets

$$
\begin{gathered}
{[U, X]=-t P, \quad[U, Y]=h P, \quad[U, Q]=t X-h Y,} \\
{[X, Y]=\left(1-\frac{h^{2}+t^{2}}{t}\right) P,} \\
{[X, Q]=-U-b P-h X-\left(1-\frac{h^{2}}{t}\right) Y,} \\
{[Y, Q]=\frac{h}{t} U+\frac{h b}{t} P-(t-1) X+h Y .}
\end{gathered}
$$

Then $W=\mu U+\lambda_{1} P+\lambda_{2} X+\lambda_{3} Y+\lambda_{4} Q \in \mathfrak{g}_{(h, t, b)}$ is a geodesic vector if and only if

$$
\left\{\begin{array}{l}
k \lambda_{4}=0, \\
\lambda_{4}\left[\left(\lambda_{3}-\mu\right) t+\lambda_{2} h+\lambda_{4} b\right]=k \lambda_{2}, \\
\left(\lambda_{4} h / t\right)\left[\left(\lambda_{3}-\mu\right) t+\lambda_{2} h+\lambda_{4} b\right]=-k \lambda_{3}, \\
\left(\lambda_{2}-\lambda_{3} h / t\right)\left(\left(\lambda_{3}-\mu\right) t+\lambda_{2} h+\lambda_{4} b\right)=-k \lambda_{1} .
\end{array}\right.
$$

For $k \neq 0$ we only have $W=0$. For $k=0$, we have the solution $\lambda_{4}=0$ and $\lambda_{2} t=\lambda_{3} h$, and the solution $\mu=t^{-1}\left(\lambda_{2} h+\lambda_{3} t+\lambda_{4} b\right)$, then the geodesic vectors $W \in$ $\mathfrak{g}_{(h, t, b)}$ have one of the following forms:

$$
\begin{aligned}
& W=\mu U+\lambda_{1} P+\left(\lambda_{3} h / t\right) X+\lambda_{3} Y, \\
& W=\frac{1}{t}\left(\lambda_{2} h+\lambda_{3} t+\lambda_{4} b\right) U+\lambda_{1} P+\lambda_{2} X+\lambda_{3} Y+\lambda_{4} Q .
\end{aligned}
$$

CASE $\mathbf{v}\left(a, b, c \in \mathbb{R}, a \neq \frac{1}{2}\right)$. The holonomy algebra $\mathfrak{h}$ is trivial and the reductive decomposition associated to $S_{(a, b, c)}$ is $\mathfrak{g}_{(a, b, c)}=\{0\}+\mathfrak{m}=\langle\{P, X, Y, Q\}\rangle$, with not always null brackets

$$
\begin{gathered}
{[X, Y]=P, \quad[X, Q]=-b P-\left(a+\frac{1}{2}\right) Y,} \\
{[Y, Q]=-c P+\left(a+\frac{1}{2}\right) X,}
\end{gathered}
$$

and $W=\lambda_{1} P+\lambda_{2} X+\lambda_{3} Y+\lambda_{4} Q \in \mathfrak{g}_{(a, b, c)}$ is a geodesic vector if and only if it satisfies (2), that is

$$
\left\{\begin{array}{l}
k \lambda_{4}=0, \\
\lambda_{4}\left[\lambda_{3}\left(a-\frac{1}{2}\right)+\lambda_{4} b\right]=k \lambda_{2}, \\
\lambda_{4}\left[\lambda_{2}\left(\frac{1}{2}-a\right)+\lambda_{4} c\right]=k \lambda_{3}, \\
\lambda_{4}\left(\lambda_{2} b+\lambda_{3} c\right)=-k \lambda_{1} .
\end{array}\right.
$$

For $k \neq 0$ we have $W=0$. For $k=0$, one solution is given by $\lambda_{4}=0$, and another solution is given by $\lambda_{2}=\lambda_{4}(a-1 / 2)^{-1} c$ and $\lambda_{3}=-\lambda_{4}(a-1 / 2)^{-1} b$, with $\lambda_{1}$ arbitrary. Then the geodesic vectors $W \in \mathfrak{g}_{(a, b, c)}$ have one of the following forms,

$$
\begin{aligned}
& W=\lambda_{1} P+\lambda_{2} X+\lambda_{3} Y, \\
& W=\lambda_{1} P+\lambda_{4}\left(a-\frac{1}{2}\right)^{-1}(c X-b Y)+\lambda_{4} Q .
\end{aligned}
$$

CASE vi $\left(a \in \mathbb{R}, a \neq \frac{1}{2}\right)$. The holonomy algebra $\mathfrak{h}$ is generated by $U$ and $V$ where $\widetilde{R}_{Q X}=\left(a^{2}-1 / 4\right) U$ and $\widetilde{R}_{Q Y}=\left(a^{2}-1 / 4\right) V$. We now consider two subcases.

SUBCASE $\mathbf{v i}_{1}$. If $a \neq-\frac{1}{2}$, the holonomy algebra $\mathfrak{h}=$ $\langle\{U, V\}\rangle$ is two-dimensional and Abelian, and the reductive decomposition associated to $S_{a}$ is $\mathfrak{g}_{a}=\mathfrak{h}+\mathfrak{m}=$ $\langle\{U, V, P, X, Y, Q\}\rangle$, with not always null brackets

$$
\begin{gathered}
{[U, X]=P, \quad[U, Q]=-X, \quad[V, Y]=P, \quad[V, Q]=-Y,} \\
{[X, Y]=2 a P, \quad[X, Q]=-\left(a^{2}-\frac{1}{4}\right) U-2 a Y,} \\
{[Y, Q]=-\left(a^{2}-\frac{1}{4}\right) V+2 a X .}
\end{gathered}
$$

(Note that if $a=0$, it is $S=0$ and the reductive decomposition corresponds to the presentation of (Os, $\left.g_{0}\right)$ as a Lorentzian symmetric space). A vector $W=\mu U+\nu V+\lambda_{1} P+\lambda_{2} X+\lambda_{3} Y+\lambda_{4} Q \in \mathfrak{g}_{a}$ is a geodesic vector if and only equation (2) is satisfied, that is,

$$
\left\{\begin{array}{l}
k \lambda_{4}=0 \\
\mu \lambda_{4}=k \lambda_{2} \\
\nu \lambda_{4}=k \lambda_{3} \\
\mu \lambda_{2}+\nu \lambda_{3}=-k \lambda_{1} .
\end{array}\right.
$$

For $k \neq 0$ we have $W_{\mathfrak{m}}=0$. For $k=0$, we have one solution given by $\mu=\nu=0$ and another solution given by $\lambda_{4}=0$ and $\mu \lambda_{2}+\nu \lambda_{3}=0$. Then the geodesic vectors $W \in \mathfrak{g}_{a}$ are of one of the following forms,

$$
\begin{aligned}
& W=\mu U+\nu V+\lambda_{1} P+\lambda_{2} X+\lambda_{3} Y, \quad\left(\mu \lambda_{2}+\nu \lambda_{3}=0\right), \\
& W=\lambda_{1} P+\lambda_{2} X+\lambda_{3} Y+\lambda_{4} Q .
\end{aligned}
$$

SubCASE $\mathbf{v i}_{2}\left(a=-\frac{1}{2}\right)$. In this case, $\widetilde{R}=0$ and $\mathfrak{h}$ is trivial, and the reductive decomposition associated to $S_{-1 / 2}$ is $\mathfrak{g}_{-1 / 2}=\{0\}+\mathfrak{m}=\langle\{P, X, Y, Q\}\rangle$, with non-null brackets

$$
[X, Y]=-P, \quad[X, Q]=Y, \quad[Y, Q]=-X .
$$

and we have that $W=\lambda_{1} P+\lambda_{2} X+\lambda_{3} Y+\lambda_{4} Q \in \mathfrak{g}_{-1 / 2}$ is a geodesic vector if and only if $k \lambda_{i}=0$ for all $i \in$ $\{1,2,3,4\}$. Then, all non-zero vectors $W \in \mathfrak{g}_{-1 / 2}$ are geodesic vectors.

Theorem III.2. For each homogeneous Lorentzian structure $S_{(a, b)}$ on $\left(\mathrm{Os}, g_{\varepsilon}\right),-1<\varepsilon<1, \varepsilon \neq 0$, given in Theorem II.5, the geodesic vectors through the origin $o \in \mathrm{Os}=G / H, G \subseteq I_{0}\left(\mathrm{Os}, g_{\varepsilon}\right)$, with the reductive decomposition $\mathfrak{g}_{(a, b)}=\mathfrak{h}+\mathfrak{m}$ associated to $S$, are of the form

$$
\begin{aligned}
& W=\lambda_{1} P+\lambda_{4} Q \\
& W=\varepsilon^{-1} \lambda_{4}\left(b-\frac{1}{2}\right) P+\lambda_{2} X+\lambda_{3} Y+\lambda_{4} Q
\end{aligned}
$$




$$
\begin{aligned}
& \text { if } a=-\varepsilon / 2 \text {, and } \\
& W=\mu U+\lambda_{1} P+\lambda_{4} Q, \\
& W=\left(a+\frac{\varepsilon}{2}\right)^{-1}\left(\lambda_{1}\left(a-\frac{\varepsilon}{2}\right)+\lambda_{4}\left(b-\frac{1}{2}\right)\right) U \\
& +\lambda_{1} P+\lambda_{2} X+\lambda_{3} Y+\lambda_{4} Q,
\end{aligned}
$$

if $a \neq-\varepsilon / 2$. Furthermore, all the geodesics are canonically homogeneous only for $(a, b)=(\varepsilon / 2,1 / 2)$.

Proof. The holonomy algebra $\mathfrak{h}$ of $\widetilde{\nabla}_{(a, b)}=\nabla-S_{(a, b)}$ is generated by the curvature operator $U=\widetilde{R}_{X Y}$ of $\widetilde{\nabla}_{(a, b)}$, given by $U(X)=-(a+\varepsilon / 2) Y, U(Y)=(a+\varepsilon / 2) X$, $U(P)=U(Q)=0$ (cf. Ref. $\left.{ }^{15}, \S 5\right)$. We must consider two cases, depending on whether the holonomy algebra $\mathfrak{h}$ is trivial or not.

CASE $a=-\varepsilon / 2$. We have $\mathfrak{h}=0$ and the reductive decomposition associated to $S_{(-\varepsilon / 2, b)}$ is $\mathfrak{g}_{(-\varepsilon / 2, b)}=\{0\}+$ $\mathfrak{m}=\langle\{P, X, Y, Q\}\rangle$, with not always null brackets given by (cf. Ref. $\left.{ }^{15}, \S 5\right)$

$$
[X, Y]=P, \quad[X, Q]=-\left(b+\frac{1}{2}\right) Y, \quad[Y, Q]=\left(b+\frac{1}{2}\right) X .
$$

A vector $W=\lambda_{1} P+\lambda_{2} X+\lambda_{3} Y+\lambda_{4} Q \in \mathfrak{g}_{(-\varepsilon / 2, b)}$ is a geodesic vector if and only if (2) holds, and this is equivalent to the following system:

$$
\left\{\begin{array}{l}
k\left(\varepsilon \lambda_{1}+\lambda_{4}\right)=0, \\
\lambda_{3}\left[-\varepsilon \lambda_{1}+\lambda_{4}\left(b-\frac{1}{2}\right)\right]=k \lambda_{2}, \\
\lambda_{2}\left[\varepsilon \lambda_{1}-\lambda_{4}\left(b-\frac{1}{2}\right)\right]=k \lambda_{3}, \\
k\left(\varepsilon \lambda_{4}+\lambda_{1}\right)=0 .
\end{array}\right.
$$

For $k \neq 0$, we only have $W=0$. For $k=0$, one solution is given by $\lambda_{2}=\lambda_{3}=0$ and another solution is given by $\varepsilon \lambda_{1}=\lambda_{4}(b-1 / 2)$. Then the geodesic vectors $W \in \mathfrak{g}_{(-\varepsilon / 2, b)}$ have one of the forms in (6).

CASE $a \neq-\varepsilon / 2$. The holonomy algebra $\mathfrak{h}$ of $\widetilde{\nabla}_{(a, b)}$ is generated by $U=\widetilde{R}_{X Y}$ and the reductive decomposition associated to $S_{(a, b)}, a \neq-\frac{\varepsilon}{2}$, is given by $\mathfrak{g}_{(a, b)}=\mathfrak{h}+\mathfrak{m}=$ $\langle\{U, P, X, Y, Q\}\rangle$, with not always null brackets given by (4) (cf. Ref. $\left.{ }^{15}, \S 5\right)$

$$
\begin{gathered}
{[U, X]=-\left(\frac{\varepsilon}{2}+a\right) Y, \quad[U, Y]=\left(\frac{\varepsilon}{2}+a\right) X,} \\
{[P, X]=\left(\frac{\varepsilon}{2}+a\right) Y, \quad[P, Y]=-\left(\frac{\varepsilon}{2}+a\right) X,} \\
{[X, Y]=U+P, \quad[X, Q]=-\left(b+\frac{1}{2}\right) Y,} \\
{[Y, Q]=\left(b+\frac{1}{2}\right) X .}
\end{gathered}
$$

Then $W=\mu U+\lambda_{1} P+\lambda_{2} X+\lambda_{3} Y+\lambda_{4} Q \in \mathfrak{g}_{(a, b)}$ is a geodesic vector if and only if it satisfies (2), that is,

$$
\left\{\begin{array}{l}
k\left(\varepsilon \lambda_{1}+\lambda_{4}\right)=0, \\
\lambda_{3}\left[-\mu(a+\varepsilon / 2)+\lambda_{1}(a-\varepsilon / 2)+\lambda_{4}(b-1 / 2)\right]=k \lambda_{2}, \\
\lambda_{2}\left[\mu(a+\varepsilon / 2)-\lambda_{1}(a-\varepsilon / 2)-\lambda_{4}(b-1 / 2)\right]=k \lambda_{3}, \\
k\left(\varepsilon \lambda_{4}+\lambda_{1}\right)=0 .
\end{array}\right.
$$

For $k \neq 0$ we only have $W_{\mathfrak{m}}=0$. For $k=0$, we have the solution given by $\lambda_{2}=\lambda_{3}=0$ and the solution given by $\mu(a+\varepsilon / 2)=\lambda_{1}(a-\varepsilon / 2)+\lambda_{4}(b-1 / 2)$. Then the geodesic vectors $W \in \mathfrak{g}_{(a, b)}$ have one of the forms in (7).

As a direct consequence of Theorems III.1 and III.2, we have the next result.

Theorem III.3. The oscillator group Os is a Lorentzian g.o. space (hence a n.g.o. space) if it is considered with each of the homogeneous presentations and reductive decompositions corresponding to the homogeneous Lorentzian structures given by:

(a) The metric $g_{0}$ and any of the following homogeneous Lorentzian structures, included in some of the cases described in Theorem II.4,

(i) $S_{(x, y, z, w)}$, for $x \neq 0, y, z, w$ not all 0 ,

(ii) $S_{(q, c)}, \quad$ for $q \neq \frac{1}{2}$,

(iii) $S_{(0,0)}$,

(iv) $S_{(h, t, b)}$, for $t \neq 0$,

(vi) $S_{a}, \quad$ for $a \neq \frac{1}{2}$.

(Note that the case $(\mathbf{v})$ does not appear).

(b) Any of the metrics $g_{\varepsilon}, \varepsilon \neq 0$, and any homogeneous Lorentzian structure $S_{(a, b)}$, such that $a \neq-\varepsilon / 2$, described in Theorem II.5.

All the Lorentzian Lie groups (Os, $\left.g_{\varepsilon}\right),-1<\varepsilon<1$, are g.o. manifolds.

Remark III.4. We recall that an important invariant of a supergravity background is the amount of preserved supersymmetry $\nu_{c}$, usually expressed as a fraction of the maximal number of supersymmetries. The authors of Ref. ${ }^{11}$ (see also Ref. ${ }^{12}$ ), based on the fact that the maximally supersymmetric backgrounds are all symmetric spaces hence homogeneous, posed themselves the question of the existence of a critical value $\nu_{c}$ which forces the background to be (at least locally) homogeneous, and study it in the context of an M-theory background $\left(M^{11}, g, F\right)$, that is, bosonic solutions of the equations of motion of eleven-dimensional supergravity. They proved that for $\nu_{c}>3 / 4$, i.e., for backgrounds admitting more than 24 supersymmetries, the Killing superalgebra acts locally transitively. The oscillator group, when endowed with suitable metrics and appropriate homogeneous Lorentzian structures, that is, those given in Theorem III.3, is thus a candidate to construct solutions (Os $\left.=G / H, g_{\varepsilon}\right) \times M^{7},-1<\varepsilon<1$, in 11-dimensional supergravity, with at least 24 of the available 32 supersymmetries.

Proposition III.5. The oscillator group (Os, $g_{0}$ ) (resp. $\left(\mathrm{Os}, g_{\varepsilon}\right),-1<\varepsilon<1, \varepsilon \neq 0$ ) admits homogeneous presentations defined by homogeneous Lorentzian structures belonging to the types specified in Table II. 


\begin{tabular}{|r|l|l|}
\hline$g_{0}$ & $S$ & Type of structure \\
\hline $\mathbf{i}$ & $S_{(x, y, z, w)}, x \neq 0$, & $\mathcal{S}_{2} \oplus \mathcal{S}_{3}$ \\
\hline ii & $S_{(q, c)}, q \neq \frac{1}{2}$ & $\begin{array}{l}\mathcal{S}_{2} \oplus \mathcal{S}_{3} \\
\mathcal{S}_{2} \quad \text { if } \quad q=-1\end{array}$ \\
\hline iii & $S_{(b, c)}$ & $\begin{array}{l}\mathcal{S}_{2} \oplus \mathcal{S}_{3} \\
\mathcal{S}_{3} \quad \text { if } \quad(b, c)=(0,0)\end{array}$ \\
\hline iv & $S_{(h, t, b)}, t \neq 0$ & $\begin{array}{l}\mathcal{S}_{2} \oplus \mathcal{S}_{3} \\
\mathcal{S}_{2} \quad \text { if } \quad h^{2}=t\left(\frac{3}{2}-t\right)\end{array}$ \\
\hline $\mathbf{v}$ & $S_{(a, b, c)}, a \neq \frac{1}{2}$ & $\begin{array}{l}\mathcal{S}_{2} \oplus \mathcal{S}_{3} \\
\mathcal{S}_{2} \quad \text { if } \quad a=-1\end{array}$ \\
\hline $\mathbf{v i}$ & $S_{a}, a \neq \frac{1}{2}$ & $\begin{array}{l}\mathcal{S}_{3} \\
\{0\} \quad \text { if } \quad a=0\end{array}$ \\
\hline \hline$g_{\varepsilon}$ & $S$ & Type of structure \\
\hline & & $\mathcal{S}_{2} \oplus \mathcal{S}_{3}$ \\
& $S_{(a, b)} \quad$ if $\quad(a, b)=(-\varepsilon,-1)$ \\
& & $\mathcal{S}_{3} \quad$ if $(a, b)=(\varepsilon / 2,1 / 2)$ \\
\hline
\end{tabular}

TABLE II. Geometric types of structures on (Os, $\left.g_{0}\right)$ and on $\left(\mathrm{Os}, g_{\varepsilon}\right),-1<\varepsilon<1, \varepsilon \neq 0$.

Proof. In the case (Os, $\left.g_{0}\right)$ we have (omitting any $S_{X_{1} X_{3} X_{2}}$ if $S_{X_{1} X_{2} X_{3}}$ already appears) that the not always null components of the structures given in Theorem II.4 are

$$
\begin{aligned}
& \left(S_{(x, y, z, w)}\right)_{P X Y}=x, \quad\left(S_{(x, y, z, w)}\right)_{Q X Y}=w+\frac{1}{2}, \\
& \left(S_{(x, y, z, w)}\right)_{X X Y}=\left(S_{(x, y, z, w)}\right)_{P Y Q}=x y, \\
& \left(S_{(x, y, z, w)}\right)_{Y Y X}=\left(S_{(x, y, z, w)}\right)_{P X Q}=-x z, \\
& \left(S_{(x, y, z, w)}\right)_{X X Q}=-\left(S_{(x, y, z, w)}\right)_{Y Y Q}=-x y z \\
& \left(S_{(x, y, z, w)}\right)_{X Y Q}=x y^{2}+\frac{1}{2}, \\
& \left(S_{(x, y, z, w)}\right)_{Y X Q}=-x z^{2}-\frac{1}{2}, \\
& \left(S_{(x, y, z, w)}\right)_{Q Q X}=z w, \quad\left(S_{(x, y, z, w)}\right)_{Q Q Y}=-y w \\
& \left(S_{(q, c)}\right)_{Q X Y}=-\left(S_{(q, c)}\right)_{Y X Q}=\frac{1}{2}, \\
& \left(S_{(q, c)}\right)_{X Y Q}=q, \quad\left(S_{(q, c)}\right)_{Q Q Y}=-c, \\
& \left(S_{(b, c)}\right)_{Q X Y}=\left(S_{(b, c)}\right)_{Y Q X}=\left(S_{(b, c)}\right)_{X Y Q}=\frac{1}{2}, \\
& \left(S_{(b, c)}\right)_{Q X Q}=b,\left(S_{(b, c)}\right)_{Q Y Q}=c, \\
& \left(S_{(h, t, b)}\right)_{Q X Y}=\frac{1}{2},\left(S_{(h, t, b)}\right)_{X X Q}=-\left(S_{(h, t, b)}\right)_{Y Y Q}=h, \\
& \left(S_{(h, t, b)}\right)_{Y X Q}=t-\frac{1}{2}, \quad\left(S_{(h, t, b)}\right)_{Q Q X}=-b, \\
& \left(S_{(h, t, b)}\right)_{X Y Q}=\frac{1}{2}-\frac{h^{2}}{t}, \quad\left(S_{(h, t, b)}\right)_{Q Y Q}=-\frac{h b}{t},
\end{aligned}
$$

$$
\begin{gathered}
\left(S_{(a, b, c)}\right)_{Q X Y}=a,\left(S_{(a, b, c)}\right)_{Q X Q}=b, \\
\left(S_{(a, b, c)}\right)_{X Y Q}=-\left(S_{(a, b, c)}\right)_{Y X Q}=\frac{1}{2}, \quad\left(S_{(a, b, c)}\right)_{Q Y Q}=c, \\
\left(S_{a}\right)_{Q X Y}=\left(S_{a}\right)_{Y Q X}=\left(S_{a}\right)_{X Y Q}=a .
\end{gathered}
$$

For each homogeneous Lorentzian structure $S$ on (Os, $\left.g_{0}\right)$ we have according to Eq. (5), that

$$
c_{12}(S)(Z)=S_{P Q Z}+S_{Q P Z}+S_{X X Z}+S_{Y Y Z},
$$

and hence all these structures are of type $\mathcal{S}_{2} \oplus \mathcal{S}_{3}$. Moreover, $\mathfrak{S}_{X_{1} X_{2} X_{3}} S_{X_{1} X_{2} X_{3}}=0$ for each $X_{1}, X_{2}, X_{3} \in \mathfrak{o s}$ when $S=S_{(q, c)}$ with $q=-1$, or $S=S_{(h, t, b)}$ if $h^{2}=t(3 / 2-t)$, or $S=S_{(a, b, c)}$ with $a=-1$, and then $S$ is of type $\mathcal{S}_{2}$ in these cases. On the other hand, $S_{(b, c)}$ is of type $\mathcal{S}_{3}$ if $(b, c)=(0,0)$. The structures $S_{a}$ are of type $\mathcal{S}_{3}$, and if $a=0$ the type is $\{0\}$.

As for $\left(\mathrm{Os}, g_{\varepsilon}\right), \varepsilon \neq 0$, by Theorem II.5 we have

$$
\begin{gathered}
\left(S_{(a, b)}\right)_{Y P X}=-\left(S_{(a, b)}\right)_{X P Y}=\frac{\varepsilon}{2}, \quad\left(S_{(a, b)}\right)_{P X Y}=a, \\
\left(S_{(a, b)}\right)_{Y X Q}=-\left(S_{(a, b)}\right)_{X Y Q}=-\frac{1}{2}, \quad\left(S_{(a, b)}\right)_{Q X Y}=b .
\end{gathered}
$$

In this case, we have

$$
\begin{aligned}
c_{12}(S)(Z)= & -\frac{1}{\sqrt{2-2 \varepsilon}} S_{P-Q, P-Q, Z}+S_{X X Z}+S_{Y Y Z} \\
& +\frac{1}{\sqrt{2+2 \varepsilon}} S_{P+Q, P+Q, Z}=0,
\end{aligned}
$$

for all the homogeneous Lorentzian structures on (Os, $g_{\varepsilon}$ ), and then each $S_{(a, b)}$ is of type $\mathcal{S}_{2} \oplus \mathcal{S}_{3}$; moreover, $S_{(a, b)}$ is of type $\mathcal{S}_{2}$ if $(a, b)=(-\varepsilon,-1)$ and it is of type $\mathcal{S}_{3}$ if $(a, b)=(\varepsilon / 2,1 / 2)$.

From Theorem III.3 and Proposition III.5 we have

Corollary III.6. The oscillator groups (Os, $\left.g_{\varepsilon}\right),-1<$ $\varepsilon<1$, admit homogeneous presentations as Lorentzian g.o. spaces defined by homogeneous Lorentzian structures, all of them belonging to the geometric type $\mathcal{S}_{2} \oplus \mathcal{S}_{3}$. Moreover, some of such structures are of type $\mathcal{S}_{2}$ (specifically, $S_{(-1, c)}$ in case $\mathbf{i i} ; S_{(h, t, b)}$ with $h^{2}=t(3 / 2-t)$ in case $\mathbf{i v}$, if $\varepsilon=0$; and $S_{(-\varepsilon,-1)}$ if $\left.\varepsilon \neq 0\right)$, and some others are of type $\mathcal{S}_{3}$ (specifically, $S_{(0,0)}$ in case $\mathbf{i i i} ; S_{a}, a \neq \pm \frac{1}{2}$, in case vi, if $\varepsilon=0$; and $S_{(\varepsilon / 2,1 / 2)}$ if $\left.\varepsilon \neq 0\right)$.

Remark III.7. We recall the results in Ref. ${ }^{12}$, $\S \S 3.4$ and Ref. ${ }^{22}$, Lem. 3 and Prop. 4, where it is proved that: (i) a reductive homogeneous Lorentzian space admitting a homogeneous Lorentzian structure of type $\mathcal{S}_{1} \oplus \mathcal{S}_{3}$ is a n.g.o. space and every null-geodesic is canonically homogeneous; (ii) a Lorentzian n.g.o. space for which every null-geodesic is canonically homogeneous admits a homogeneous Lorentzian structure of type $\mathcal{S}_{1} \oplus \mathcal{S}_{3}$. So, as pointed out by Meessen ${ }^{22}$ (p. 212), homogeneous Lorentzian spaces admitting a homogeneous Lorentzian structure of type $\mathcal{S}_{1} \oplus \mathcal{S}_{3}$ play the same rôle in the 
Lorentzian n.g.o. spaces as that played by naturally reductive spaces in the class of Lorentzian g.o. spaces (cf. Ref. ${ }^{16}$, Ch. X, §3). Theorems III.1 and III.2 above thus tell us that:

(a) Any geodesic in $\left(\mathrm{Os}, g_{0}\right)$ is canonically homogeneous whenever the space is considered with the homogeneous description defined by any of the structures

$S_{\lambda}=\lambda(\xi \otimes(\alpha \wedge \beta)-\beta \otimes(\alpha \wedge \xi)+\alpha \otimes(\beta \wedge \xi)), \quad \lambda \in \mathbb{R}$,

which are of type $\mathcal{S}_{3}$ (for $\lambda=0$ the type is $\{0\}$, that is, the space is Lorentzian symmetric).

(b) Any geodesic in $\left(\mathrm{Os}, g_{\varepsilon}\right)$ is canonically homogeneous whenever the space is considered with the homogeneous description defined by the structure

$$
\begin{aligned}
& S_{\left(\frac{\varepsilon}{2}, \frac{1}{2}\right)}=\frac{1}{2}(\varepsilon \beta \otimes(\eta \wedge \alpha)-\varepsilon \alpha \otimes(\eta \wedge \beta)-\beta \otimes(\alpha \wedge \xi) \\
&+\alpha \otimes(\beta \wedge \xi)+(\varepsilon \eta+\xi) \otimes(\alpha \wedge \beta)),
\end{aligned}
$$

which is of type $\mathcal{S}_{3}$.

\section{Acknowledgements}

The three authors have been partially supported by the Ministry of Economy and Competitiveness, Spain, under Project MTM2011-22528.

${ }^{1}$ Ambrose, W., and Singer, I.M., "On homogeneous Riemannian manifolds," Duke Math. J. 25, 647 (1958).

${ }^{2}$ Boucetta, M., and Medina, A., "Solutions of the Yang-Baxter equations on quadratic Lie groups: The case of oscillator groups," J. Geom. Phys. 61, 2309 (2011).

${ }^{3}$ Bromberg, S., and Medina, A., "A homogeneous spacetime model with singularities," J. Math. Phys. 41, 8190 (2000).

${ }^{4}$ Bromberg, S., and Medina, A., "Geometry of oscillator groups and locally symmetric manifolds," Geom. Dedicata 106, 97 (2004).

${ }^{5}$ Calvaruso, G., Kowalski, O., and Marinosci, R.A., "Homogeneous geodesics in solvable Lie groups," Acta Math. Hungar. 101, 313 (2003).

${ }^{6}$ Cartan, É., "Sur une classe remarquable d'espaces de Riemann," Bull. Soc. Math. France, 55, 114 (1927).

${ }^{7}$ Console, S., Ovando, G.P., and Subils, M., "Solvable models for Kodaira surfaces," preprint, 2011. arXiv:1111.2417v1.
${ }^{8}$ Durán Díaz, R., Gadea, P.M., and Oubiña, J.A., "Reductive decompositions and Einstein-Yang-Mills equations associated to the oscillator group," J. Math. Phys. 40, 3490 (1999).

${ }^{9}$ Dušek Z., "Survey on homogeneous geodesics," Note Mat. 1, suppl. no. 1, 147 (2008).

${ }^{10}$ Dušek, Z., Kowalski, O., and Nikcevic, S. Ž., "New examples of Riemannian g.o. manifolds in dimension 7," Differential Geom. Appl. 21, 65 (2004).

${ }^{11}$ Figueroa-O'Farrill, J., Meessen, P., and Philip, S., "Supersymmetry and homogeneity of M-theory backgrounds," Classical Quantum Gravity 22, 207 (2005).

${ }^{12}$ Figueroa-O'Farrill, J., Meessen, P., and Philip, S., "Homogeneity and plane-wave limits," J. High Energy Phys. 050, no. 5, 42 pp. (2005).

${ }^{13}$ Gadea, P.M., and Oubiña, J.A., "Homogeneous pseudo-Riemannian structures and homogeneous almost para-Hermitian structures," Houston J. Math. 18, 449 (1992).

${ }^{14}$ Gadea, P.M., and Oubiña, J.A., "Reductive homogeneous pseudo-Riemannian manifolds," Monats. Math. 124, 17 (1997).

${ }^{15}$ Gadea, P.M., and Oubiña, J.A., "Homogeneous Lorentzian structures on the oscillator groups," Arch. Math. 73, 311 (1999).

${ }^{16}$ Kobayashi, S., Nomizu, K., Foundations of Differential Geometry, vol. II, (Wiley, 1969).

${ }^{17}$ Levichev, A.V., "Chronogeometry of an electromagnetic wave given by a biinvariant metric on the oscillator group," Siberian Math. J. 27, 237 (1986).

${ }^{18}$ Levichev, A.V., "Methods of investigation of the causal structure of homogeneous Lorentz manifolds," Siberian Math. J. 31, 395 (1990).

${ }^{19}$ Medina, A., "Groupes de Lie munis de métriques bi-invariantes," Tohôku Math. J. 37, 81 (1985).

${ }^{20}$ Medina, A., and Revoy, P., "Les groupes oscillateurs et leurs réseaux," Manuscripta Math. 52, 405 (1985).

${ }^{21}$ Meessen, P., "Homogeneous Lorentzian spaces admitting a homogeneous structure of type $\mathcal{T}_{1} \oplus \mathcal{T}_{3}$, J J. Geom. Phys. 56, 754 (2006).

${ }^{22}$ Meessen, P., "Homogeneous Lorentzian spaces whose nullgeodesics are canonically homogeneous," Lett. Math. Phys. 75, 209 (2006).

${ }^{23}$ Nappi, Ch., and Witten, E., "Wess-Zumino-Witten model based on a nonsemisimple group," Phys. Rev. Lett. 71, 3751 (1993).

${ }^{24}$ Nomizu, K., "Invariant affine connections on homogeneous spaces," Amer. J. Math. 76, 33 (1954).

${ }^{25}$ Philip, S., "Penrose limits of homogeneous spaces," J. Geom. Phys. 56, 1516 (2006).

${ }^{26}$ Streater, R.F., "The representations of the oscillator group," Comm. Math. Phys. 4, 217 (1967).

${ }^{27}$ Tricerri, F., and Vanhecke, L., Homogeneous structures on Riemannian manifolds, London Math. Soc. Lect. Note Ser., 83, (Cambridge Univ. Press, 1983). 